\title{
Broad host range plasmids can invade an unexpectedly diverse fraction of a soil bacterial community
}

\author{
Uli Klümper ${ }^{1}$, Leise Riber ${ }^{2}$, Arnaud Dechesne ${ }^{1}$, Analia Sannazzarro², Lars H Hansen ${ }^{2,3}$, \\ Søren J Sørensen ${ }^{2}$ and Barth F Smets ${ }^{1}$ \\ ${ }^{1}$ Department of Environmental Engineering, Technical University of Denmark, Kgs. Lyngby, Denmark; \\ ${ }^{2}$ Section for Microbiology, Department of Biology, University of Copenhagen, Copenhagen, Denmark and \\ ${ }^{3}$ Department of Environmental Science, Aarhus University, Roskilde, Denmark
}

\begin{abstract}
Conjugal plasmids can provide microbes with full complements of new genes and constitute potent vehicles for horizontal gene transfer. Conjugal plasmid transfer is deemed responsible for the rapid spread of antibiotic resistance among microbes. While broad host range plasmids are known to transfer to diverse hosts in pure culture, the extent of their ability to transfer in the complex bacterial communities present in most habitats has not been comprehensively studied. Here, we isolated and characterized transconjugants with a degree of sensitivity not previously realized to investigate the transfer range of IncP- and IncPromA-type broad host range plasmids from three proteobacterial donors to a soil bacterial community. We identified transfer to many different recipients belonging to 11 different bacterial phyla. The prevalence of transconjugants belonging to diverse Gram-positive Firmicutes and Actinobacteria suggests that inter-Gram plasmid transfer of IncP-1 and IncPromAtype plasmids is a frequent phenomenon. While the plasmid receiving fractions of the community were both plasmid- and donor- dependent, we identified a core super-permissive fraction that could take up different plasmids from diverse donor strains. This fraction, comprising $80 \%$ of the identified transconjugants, thus has the potential to dominate IncP- and IncPromA-type plasmid transfer in soil. Our results demonstrate that these broad host range plasmids have a hitherto unrecognized potential to transfer readily to very diverse bacteria and can, therefore, directly connect large proportions of the soil bacterial gene pool. This finding reinforces the evolutionary and medical significances of these plasmids.
\end{abstract}

The ISME Journal (2015) 9, 934-945; doi:10.1038/ismej.2014.191; published online 21 October 2014

\section{Introduction}

Conjugal plasmid transfer is a process by which bacteria horizontally transfer complete sets of genes to other, potentially distantly related, organisms. Conjugal plasmids frequently carry accessory genes, often encoding antibiotic or metal resistances, catabolic pathways or virulence factors. They are often implicated in the evolution of pathogenic bacteria and the rapid spread of antibiotic resistance, likely fostering the rise of multiple-resistant microbes in hospitals (Levy and Marshall, 2004) and animal husbandries (Zhu et al., 2013). Although the relevance of plasmid transfer has become very acute in this age of massive antibiotic usage, plasmids have been exchanged for much longer, and many

Correspondence: BF Smets, Technical University of Denmark, Department of Environmental Engineering, Miljøvej 113, 2840 Kgs. Lyngby, Denmark.

E-mail bfsm@env.dtu.dk

Received 6 July 2014; revised 17 August 2014; accepted 21 August 2014; published online 21 October 2014 prokaryotic genomes present signs of intense past horizontal gene transfer (Ochman et al., 2000).

Plasmids present different abilities to transfer into, and be maintained in, distantly related bacterial hosts and are loosely categorized as having a narrow or broad host range. The transfer of narrow host range plasmids is limited at one of the steps required for successful transfer, such as the formation of mating pairs, the avoidance of the recipient's restriction system or the correct expression of its replication and maintenance systems in the recipient (Thomas and Nielsen, 2005). Some broad host range plasmids can transfer across bacterial phyla and even across domains of life (Heinemann and Sprague, 1989; Waters, 2001), and several genetic determinants conferring broad host transfer capability have been identified (Jain and Srivastava, 2013).

The host range is thus a key parameter that controls the ecology and fate of plasmids. The evaluation of host range has traditionally been conducted using few individual pure strains as recipients, a situation that contrasts with the fact that most bacteria-and thus most plasmids-exist 
within complex communities of hundreds to thousands of species (Hong et al., 2006; Brown Kav et al., 2012). Among these diverse communities, all strains are obviously not equally permissive toward plasmid receipt, even for broad host range plasmids. This notion was supported when studying plasmid transfer to a range of strains isolated from marine water or wastewater treatment bioreactors (Sørensen, 1993; Inoue et al., 2005). With the use of fluorescent reporter genes to track plasmids, which reduces the need for selection and cultivation steps to identify transconjugants, it has become apparent that, in complex communities, broad host range plasmids can indeed be received by bacteria distantly related to the donor, even in the absence of selective pressure for plasmid carriage (De Gelder et al., 2005; Musovic et al., 2006, 2014; Shintani et al., 2014). However, these efforts, limited to inspection of a few hundred transconjugants at best, most likely underestimate the true diversity of transconjugal pools and do not accurately describe how plasmid permissiveness may vary across taxa in complex microbial communities.

Horizontal gene transfer between different species has been recognized as a common and major evolutionary process (Zhaxybayeva and Doolittle, 2011), most acutely demonstrated in the heavy interconnection between the resistome of soil dwelling bacteria and human pathogens (Forsberg et al., 2012). The behavior of this environmental resistome may, thus, govern the spread of antibiotic resistance genes to pathogens (Finley et al., 2013). Plasmids serve as main vessels of gene flow in microbial communities, linking distinct genetic pools (Norman et al., 2009; Halary et al., 2010). The in situ host range of plasmids may, then, well govern the taxonomic breadth across which gene flow occurs.

Here, taking advantage of high-throughput cell sorting and next-generation sequencing technologies, we map for the first time the intrinsic diversity of the bacterial recipients of broad host range plasmids in a microbial community extracted from soil, under conditions where cell-to-cell contacts are maximized. We analyzed matings initiated with combinations of three plasmid donors and three plasmids to identify how permissiveness toward broad host range plasmids is distributed across taxa among the recipient community.

\section{Materials and methods}

Donor strain construction

Soil bacterial communities were challenged with various plasmid-donor combinations through solid surface filter matings. The plasmids were marked with a genetic tag encoding conditionally expressible green fluorescent proteins (GFPs). The used entranceposon (Bahl et al., 2009) carries a $\operatorname{lacl}^{q}$ repressible promoter upstream the gfpmut3 gene, encoding for the GFP. Plasmid donor strains were all chromosomally tagged with a gene cassette encoding constitutive red fluorescence and constitutive $\operatorname{lacI}^{q}$ production. As a result, there is no $g f p$ expression in the donor strains, but upon plasmid transfer to a soil bacterium, $g f p$ expression is possible, resulting in green fluorescent cells or microcolonies, which can be detected and sorted by fluorescence microscopy or fluorescent activated cell sorting (FACS), respectively (Figure 1) (Sørensen et al., 2005). Pseudomonas putida KT2440, Escherichia coli MG1655 and Kluyvera sp. served as donor strains, and were each electroporated with the plasmid pGRG36-lacI ${ }^{q}-p L p p$ $m$ Cherry- $K m^{R}$ carrying both the transposase genes and the Tn7 lacI ${ }^{q}-p L p p-m C h e r r y-K m^{R}$ region for specific integration of the lacI ${ }^{q}-p L p p$-mCherry-Km ${ }^{R}$ gene cassette into the chromosomal attTn7 site. Colonies were selected for $\mathrm{Km}^{\mathrm{R}}$ on Luria-Bertani (LB) agar plates at $30^{\circ} \mathrm{C}$. Colonies were restreaked on selective $\mathrm{LB}$ agar plates at $30^{\circ} \mathrm{C}$, incubated in liquid LB overnight culture without antibiotics at $30^{\circ} \mathrm{C}$ and finally streaked on LB agar plates without selection at $37^{\circ} \mathrm{C}$ for integration of the gene cassette and subsequent loss of the Tn7 helper plasmid. Colonies were tested for successful loss of helper plasmid and chromosomal integration of gene cassette by PCR (McKenzie and Craig, 2006). The same colonies were also phenotypically verified to be bright red fluorescent using stereomicroscopy.

\section{Construction of gfpmut3-tagged plasmid pKJK5}

Plasmids RP4 and pIPO2tet have been constructed earlier (Musovic et al., 2010, 2014). The $54 \mathrm{kbp} \mathrm{IncP-1}$

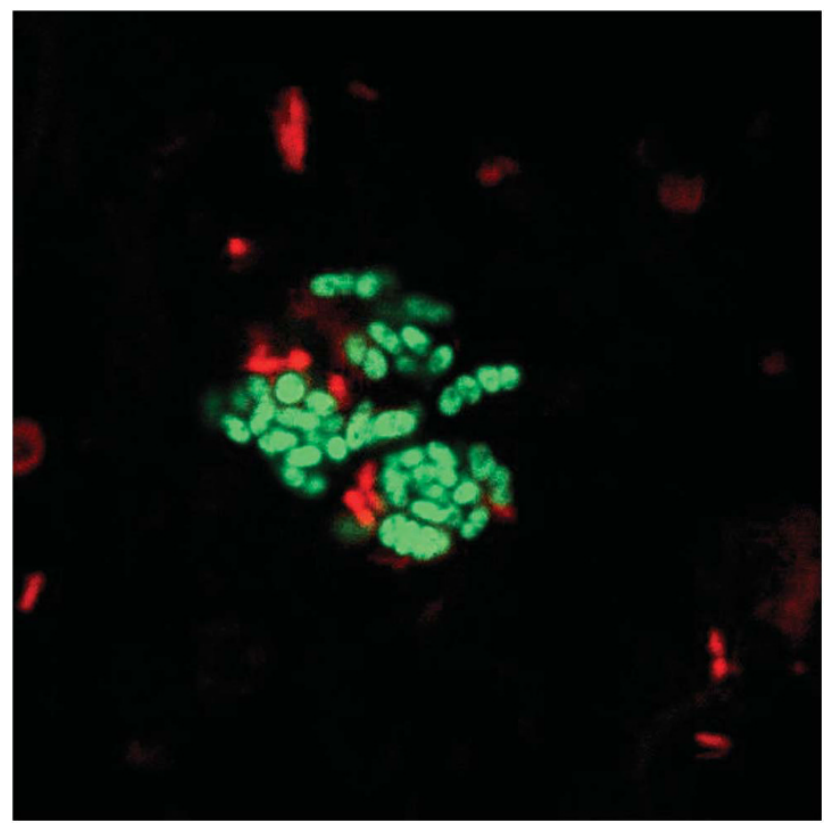

Figure 1 Typical transconjugal microcolonies for plasmid pKJK5::gfp introduced through E. coli MG1655::lacI $I^{q} p L p p-$ $m$ Cherry-Km$m^{R}$. Observation was carried out with a confocal laser scanning microscope (CLSM). Transconjugants are green fluorescent because of $g f p$ expression, $g f p$-repressed donor cells are red fluorescent through chromosomal mCherry tagging, black background represents soil bacteria. 
plasmid, pKJK5, originally isolated from a soil/ manure environment, harbors a tetracycline and a trimethoprim resistance determinant, and a class 1 integron (Sengeløv et al., 2001). The entranceposon $\left[\mathrm{Km}^{\mathrm{R}}\right.$, PA10403-gfpmut3], carrying a kanamycin resistance determinant and a lacI $^{q}$ repressible promoter upstream the gfpmut3 gene, encoding $g f p$, was derived from $\mathrm{pEntranceposon}\left[\mathrm{Km}^{R}\right]$ (Finnzymes, Thermo Scientific, Waltham, MA, USA; F-766) and randomly inserted into the plasmid pKJK5 using the artificial $\mathrm{Mu}$ transposon in vitro delivery system as described previously (Bahl et al., 2009). Transformed Escherichia coli GeneHogs single colonies were selected for resistance toward trimethoprim and kanamycin and screened for sensitivity toward tetracycline to select for plasmid derivatives with an entranceposon insert location directed to an accessory element (the tetracycline resistance determinant), thereby excluding any potential impacts on conjugation transfer ability. The exact insert location of $\left[\mathrm{Km}^{R}\right.$, PA10403gfpmut3] in the selected pKJK5 derivative of this study was determined by sequencing from the inserted fragment in one direction using primer Seq_Bw_Ent_gfp: 5'-GCCAGAACCGTTATGATGTC GG-3'. The insertion mapped to position $30.614 \mathrm{bp}$ in the tetA gene (30.435-31.634 bp) of plasmid pKJK5 (accession no. AM261282). The selected gfpmut3-tagged pKJK5 plasmid was finally introduced into E. coli MG1655::lacI ${ }^{q}-p L p p-m C h e r r y-$ $\mathrm{Km}^{R}$, P. putida KT2440::lacI ${ }^{q}$-pLpp-mCherry-Km ${ }^{R}$ and Kluyvera sp.::lacI ${ }^{q}$-pLpp-mCherry-Km${ }^{R}$ cells by transformation.

\section{Soil sampling and community extraction}

Soil samples were taken at the annually tilled CRUCIAL (Closing the Rural Urban Nutrient Cycle) agricultural field site (Taastrup, Denmark) from a plot subjected to no further agricultural treatment (Magid et al., 2006). Soil samples were collected in late fall 2012. Samples were taken from three different plots of the treatment. Each plot was sampled for $1 \mathrm{~kg}$ of soil at five locations. The resulting soil volume was sieved and homogenized to obtain a representative sample. From a total of $30 \mathrm{~g}$ of the homogenized chosen soils, indigenous bacterial communities were isolated by Nycodenz extraction (Musovic et al., 2010) and used as recipients in the mating assay. Donor strains were grown overnight in LB medium supplemented with the plasmid-specific antibiotics (Table 1) and were harvested by centrifugation.

\section{Solid surface filter mating assay}

The extracted recipient community was challenged with exogenous plasmids via solid surface filter matings (Musovic et al., 2010) modified to an initial ratio of donor-to-recipient bacteria of 1:1 at a density of $\sim 30000$ bacteria $\mathrm{mm}^{-2}$ on the filter. As a growth medium, we used a $10 \%$ soil extract medium as described by Musovic et al. (2010) buffered at pH 7.2 with $5 \mathrm{~mm} 3$-(N-morpholino)propanesulfonic acid and supplemented with $20 \mu \mathrm{g} \mathrm{ml}^{-1}$ nystatin to avoid fungal growth. Unlike in Musovic et al. (2010), we did not use additional nutrient additions, but only relied on soil-extracted nutrients to support activity during the mating incubations. Successful conjugation was checked after $48 \mathrm{~h}$ by epifluorescence stereomicroscopy and confocal laser scanning microscopy (Figure 1) (Musovic et al., 2010).

\section{Cell collection and triple-gated FACS of \\ transconjugants}

Cells from five filters per mating combination and replicate were harvested in $2 \mathrm{ml}$ of $0.9 \% \mathrm{NaCl}$ solution by vortexing for $3 \mathrm{~min}$. Flow cytometric detection of cells was carried out using a FACSAria IIIu (Becton Dickinson Biosciences, San Jose, CA, USA). The following settings and voltages were used during analysis: forward scatter $=505 \mathrm{~V}$, side scatter $=308 \mathrm{~V}$ and detectors for green (bandpass filter $530 / 30 \mathrm{~nm}$ ) and red fluorescence (bandpass filter $610 / 20 \mathrm{~nm}$ ) were set at 508 and $500 \mathrm{~V}$, respectively. A $70 \mu \mathrm{m}$ nozzle was used at a sheath fluid pressure of $70 \mathrm{psi}$. The BD FACSDiva software v.6.1.3 was used for both operating and analyzing results. Sorting was performed using a $488 \mathrm{~nm}$ $(20 \mathrm{~mW})$ laser connected to the green fluorescence detector at $515-545 \mathrm{~nm}$ and a $561 \mathrm{~nm}(50 \mathrm{~mW})$ laser connected to the red fluorescence detector at $600-$ $620 \mathrm{~nm}$. Three gates were defined in bivariate plots to sort for transconjugants. On the side scatter-A vs forward scatter-A plot, a gate for only particles of bacterial size was used. On the FITC-A vs side scatter -A plot, a gate was set that covered all green fluorescent particles, while using an additional non-red gate on the PE-Texas Red-A vs side scatterA plot excluded all small autofluorescent particles from soil or leaking donors (Figure 2) to sort out only transconjugants. All samples were diluted in $0.9 \% \mathrm{NaCl}$ to $\sim 2000$ counting events $\mathrm{s}^{-1}$ before FACS to assure for optimal sorting. Transconjugants that originally made up for $<0.1 \%$ of the total community in the filter matings and were enriched to up to $82 \%$ in a first fast sorting step, before isolating over 10000 transconjugants per sample in a second purification step, leading to $100 \%$ purity of green cells as observed by fluorescent counting in the flow cytometer. Plating of more than 200 isolated transconjugants on $10 \%$ soil extract medium (Musovic et al., 2010) resulted in detection of green fluorescence in all colonies, additionally verifying purification of $g f p$-expressing transconjugants. Of the isolated transconjugants, 20 were subject to $16 \mathrm{~S}$ rRNA gene sequences; the recovery of proteobacterial, sphingobacterial and actinobacterial phylotypes indicated diversity among transconjugants. 
Table 1 Plasmids and donor strains used in this study

\begin{tabular}{|c|c|c|c|c|}
\hline Donor & \multicolumn{3}{|c|}{ Chromosomal marker } & Reference \\
\hline $\begin{array}{l}\text { Pseudomonas putio } \\
\text { Escherichia coli MC } \\
\text { Kluyvera sp. }\end{array}$ & \multicolumn{3}{|c|}{$\begin{array}{l}\text { lacI } I^{q}-p L p p-m C h e r r y-K m^{R} \\
\text { lacI } I^{q}-p L p p-m C h e r r y-K m^{R} \\
\text { lacI }{ }^{q}-p L p p-m C h e r r y-K m^{R}\end{array}$} & $\begin{array}{l}\text { This study } \\
\text { This study } \\
\text { This study }\end{array}$ \\
\hline Plasmid & Inc group & Resistance conferred & Host range & Reference \\
\hline $\begin{array}{l}\text { RP4::Plac::gfp } \\
\text { pIPO2tet::Plac::gfp } \\
\text { pKJK5::Plac::gfp }\end{array}$ & $\begin{array}{l}\text { IncP-1 } \alpha \\
\text { IncPromA } \\
\text { IncP-1 } \varepsilon\end{array}$ & $\begin{array}{l}\operatorname{Tet}^{\mathrm{R}}, \mathrm{Amp}^{\mathrm{R}}, \mathrm{Km}^{\mathrm{R}} \\
\mathrm{Tet}^{\mathrm{R}} \\
\operatorname{Tmp}^{\mathrm{R}} \mathrm{Tet}^{\mathrm{R}}\end{array}$ & $\begin{array}{l}\text { Broad } \\
\text { Broad } \\
\text { Broad }\end{array}$ & $\begin{array}{l}\text { (Musovic et al., 2010) } \\
\text { (Musovic et al., 2014) } \\
\text { This study }\end{array}$ \\
\hline
\end{tabular}

Bacterial cell lysis, amplification and sequencing Bacterial transconjugal cells from the second sort, initially collected in $5 \mathrm{ml}$ sterile polystyrene round-bottom Falcon tubes (BD Biosciences, San Jose, CA, USA) with $0.5 \mathrm{ml}$ of $0.9 \% \mathrm{NaCl}$ solution, were transferred to $1.5 \mathrm{ml}$ Eppendorf tubes and centrifuged at $10000 \mathrm{~g}$ for $30 \mathrm{~min}$ to collect the cell pellets. The supernatant was carefully removed, the cell pellet suspended in $20 \mu \mathrm{l}$ of Lyse and Go PCR Reagent (Thermo Scientific) and the lysis mixtures transferred to $0.2 \mathrm{ml}$ amplification tubes. Cell lysis was subsequently performed in an Arktik Thermal Cycler (Thermo Scientific) using the program: one initial step at $57^{\circ} \mathrm{C}$ for $30 \mathrm{~s}$, a second step at $8{ }^{\circ} \mathrm{C}$ for $30 \mathrm{~s}$, a third step at $65{ }^{\circ} \mathrm{C}$ for $90 \mathrm{~s}$, a fourth step with heating to $97^{\circ} \mathrm{C}$ for $3 \mathrm{~min}$, a fifth step with cooling to $8^{\circ} \mathrm{C}$ for $60 \mathrm{~s}$, a sixth step with heating to $65^{\circ} \mathrm{C}$ for $3 \mathrm{~min}$ followed by additional heating to $97^{\circ} \mathrm{C}$ for $60 \mathrm{~s}$ and cooling to $65^{\circ} \mathrm{C}$ for $60 \mathrm{~s}$ with a final end step at $80{ }^{\circ} \mathrm{C}$. DNA-containing cell lysis products were immediately put on ice and used directly for subsequent PCR. Then, $5 \mu \mathrm{l}$ of the cell lysis product from the previous step were used for sequencing library preparation. Tag-encoded $16 \mathrm{~S}$ rRNA gene pyrosequencing was carried out after amplification of the $\mathrm{V} 3$ and $\mathrm{V} 4$ region (primers: 341F, 5'-CCTAYGGGRBGCASCAG-3 and 806R, 5'GGACTACNNGGGTATCTAAT-3) using the PCR procedures and GS FLX Titanium chemistry as described previously (Hansen et al., 2012).

\section{Sequence analysis and tree construction}

Sequence analysis was carried out using Mothur v.1.32.1 (Schloss et al., 2009) and the 454 SOP (Schloss et al., 2011) as accessed on 11 January 2013 on http://www.mothur.org/wiki/454_SOP. Sequences were classified based on the RDP (Ribosomal Database Project) classifier (Wang et al., 2007). Phylogenetic trees were constructed using iToL (http://itol.embl.de/) (Letunic and Bork, 2007). All sequences have been submitted to the European Nucleotide Archive and can be accessed under study accession number PRJEB7443.

\section{Results and discussion}

High-throughput isolation and sequencing of transconjugants

We explored the ability of a bacterial community extracted from soil to engage in horizontal gene transfer and receive one of three $g f p$-tagged broad host range plasmids from three different red fluorescent-tagged donor strains in which plasmidmediated gfp expression is repressed (Table 1). In soil, physical barriers limit contact between freshly introduced plasmid donors and potential recipients (Dechesne et al., 2005); here we maximized cell-tocell contact in a gene transfer assay (Musovic et al., 2010) to study the intrinsic permissiveness of the recipient community. All three plasmids (RP4, pIPO2tet and pKJK5) were introduced to the soil community in matings with a Pseudomonas putida donor strain, whereas plasmid pKJK5 was also introduced via E. coli and Kluyvera sp. donors (Supplementary Table 1). After mating, the $g f p$ expressing transconjugant cells (Figure 1) were isolated from the mixed community by FACS. A novel triple-gated FACS approach based on size, green fluorescence and lack of red fluorescence allowed specific isolation of large numbers of transconjugant cells, in spite of their low relative abundance $(<0.1 \%)$ in the mating mixture (Figure 2$)$. At least 14000 transconjugant cells were obtained for each mating replicate, corresponding to $28000-$ 116500 transconjugants per donor-plasmid combination, depending on the number of replicate matings. The 11 pools of sorted transconjugants as well as the total soil recipient community were then subjected to deep amplicon sequencing of $16 \mathrm{~S}$ rRNA genes, resulting in 29894-50398 sequences per sample after processing with the Mothur pipeline (Schloss et al., 2009). This corresponds to more sequences than sorted transconjugants for most samples (Supplementary Table 1), providing an adequate picture of the observed plasmid transfer range.

Transconjugal pools are plasmid-and donor-specific The phylogenetic structure of the transconjugal pools was compared after clustering the partial 16S 


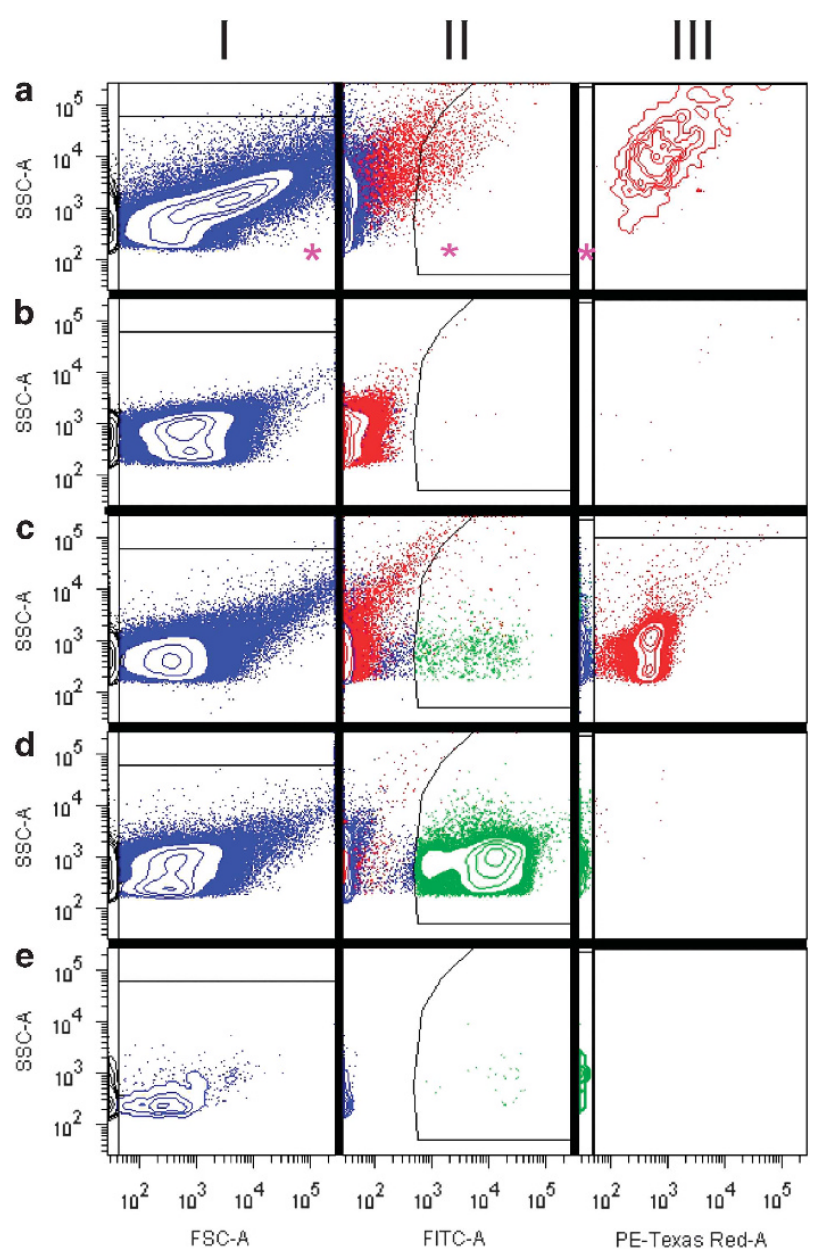

Figure 2 FACS sorting of transconjugal cells from a mating mixture initiated with soil bacteria and E. coli carrying pKJK5. The procedure consists in three successive gates (marked by pink stars in panel a). Gate I sorts for bacterial size based on forward and side scatter (SSC); Gate II sorts for green fluorescent cells; and Gate III selects only those green cells that display no red fluorescence. Line a shows the sorting of the initial soil bacterial recipient community in the absence of any donor strain and proves that the presence of green autofluorescent particles (a-II) does not yield false positives as they are excluded at the third gate, because of their red fluorescence (a-III). The sorting of a pure culture of the donor strain is shown in panel (b), where, again, no false-positive events are recorded at the final gate. Panel (c) represents the analysis of the mating mixture before sorting. Panel (d) shows the enrichment of transconjugants after the first fast enrichment sorting step to over $80 \%$ transconjugal cells, with minor contamination by donor or soil particles. Panel (e) shows how only pure transconjugants are obtained after the second purification sorting step.

rRNA gene sequences in operational taxonomic units (OTUs) at 97\% similarity. The 11 transconjugal pools clustered clearly and significantly apart from the recipient community, as shown by principal coordinate analysis (Figure 3) and analysis of molecular variance (Excoffier et al., 1992) $(P=0.028)$. Mating plates contained soil extracts as nutrient sources and growth on filter did not significantly modify the soil community structure $(P=0.797)$ based on UNIFRAC comparisons (Lozupone et al., 2011), in spite of a diversity

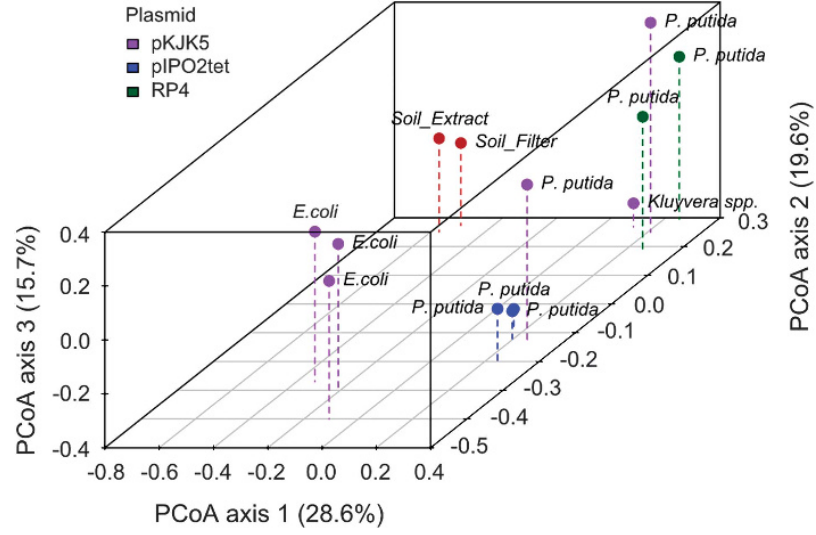

Figure 3 Principal coordinate analysis (PCoA) of individual transconjugal pools, as well as of the extracted soil community (Soil_Extract) and the reference soil community as grown on filters (Soil Filter) based on the ThetaYC algorithm (Yue and Clayton, 2005). Each axis explains a certain fraction of dissimilarity according to the axis loading given within parentheses. The three different plasmids are represented by color. The three different donor strains are named next to the data points.

reduction by $72 \%$. The transconjugal pools were clearly distinct from the recipient community, and also differed from each other based on plasmid or donor. Considering different plasmids in an identical donor strain ( $P$. putida) and providing the same plasmid (pKJK5) in different donor strains revealed phylogenetically distinct transconjugal pools (analysis of molecular variance, $P<0.001$ ). Hence, plasmid acquisition is not a stochastic process, even for broad host range plasmids. Although replicates of the same donor-plasmid combinations differed based on weighted UNIFRAC comparisons $(P<0.05)$, the average interreplicate dissimilarity $(W=0.36)$ was clearly less than the dissimilarity between different plasmid-donor combinations $(W=0.49)$ or between transconjugal pools and the soil community $(W=0.60)$. Slight differences between the replicates can also be seen in the phylum level distribution of transconjugants (Supplementary Figure 1). This dissimilarity between replicates can most likely be decreased through sorting of higher numbers of transconjugants per replicate, as replicates from the same donor-plasmid combinations grouped significantly together in principal coordinate analysis $(P<0.01)$ (Figure 3). Based on this principal coordinate analysis grouping and because the number of replicates per combination differed (Supplementary Table 1), replicates were pooled for subsequent phylogenetic analysis.

Transconjugal pools span most of the major bacterial phyla

More than 300 transconjugant OTUs were detected across all plasmid-donor combinations (Figures 4 and 5), a large expansion over the low number of distinct bacterial isolates identified previously from matings in complex environmental communities (De Gelder et al., 2005; Musovic et al., 2014, 2010; 


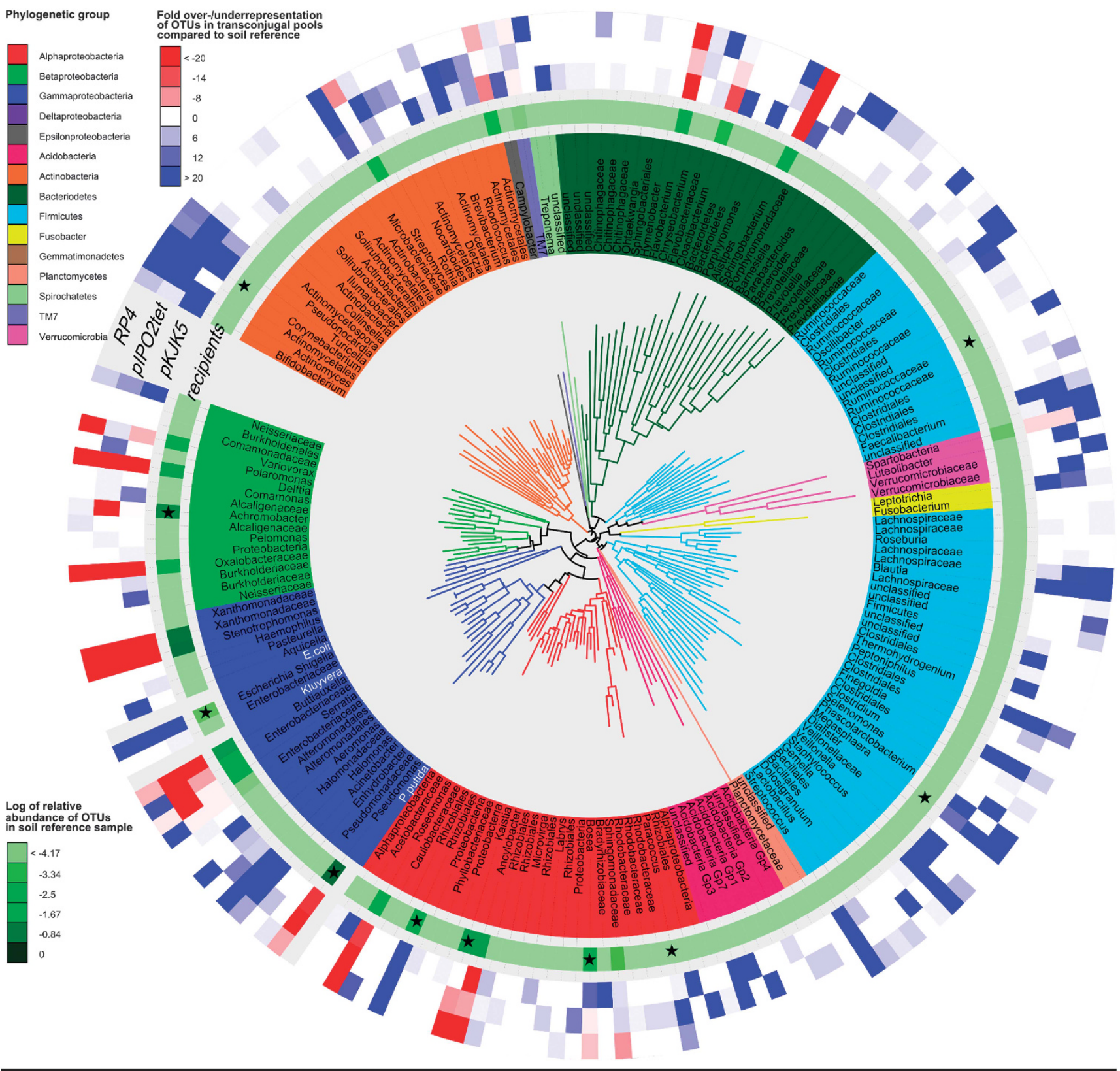

Figure 4 Phylogenetic tree showing all identified transconjugant OTUs for three different plasmids (pKJK5, RP4 and pIPO2tet) from the same donor (P. putida). The colors of the branches mark different phylogenetic groups. The three donor strains are shown in white letters in the trees. One green heatmap circle around the tree represents the log-transformed relative OTU abundance in the soil referencerecipient community. Three heatmap circles in blue and red display the $x$-fold over- and underrepresentation of the OTU in the respective transconjugal pool in comparison with the abundance in the reference soil sample. Stars mark the shared (present in all three transconjugal pools) and abundant (present at more than 1\% relative sequence abundance) transconjugant OTUs, which constitute the core super-permissive community fraction. Sample size was normalized to 30000 sequences per transconjugal pool.

Shintani et al., 2014). As expected, Proteobacteria, known to be the main hosts for the studied broad host range plasmids (Suzuki et al., 2010), were represented. Unlike in previous studies (Musovic et al., 2010; Shintani et al., 2014), all five classes $(\alpha-\varepsilon)$ of Proteobacteria were identified among the transconjugants. More strikingly, the diversity of transconjugants extended much beyond the proteobacterial phylum, and included diverse members of 10 additional phyla including Verrucomicrobia, Bacteroidetes and Actinobacteria, some of which are known as poorly cultivable (Joseph et al., 2003). The IncP transfer apparatus is known to build conjugative bridges between a huge variety of organisms (Grahn et al., 2000; Thomas and Nielsen, 2005). Shuttle vectors for gene transfer from Proteobacteria to distantly related recipients such as Cyanobacteria (Wolk et al., 1984) or Grampositive bacteria and yeast (Heinemann and Sprague, 1989; Samuels et al., 2000) have, indeed, been built using the RP4 transfer system, an IncP- $1 \alpha$ subgroup plasmid. Although the wide transfer 


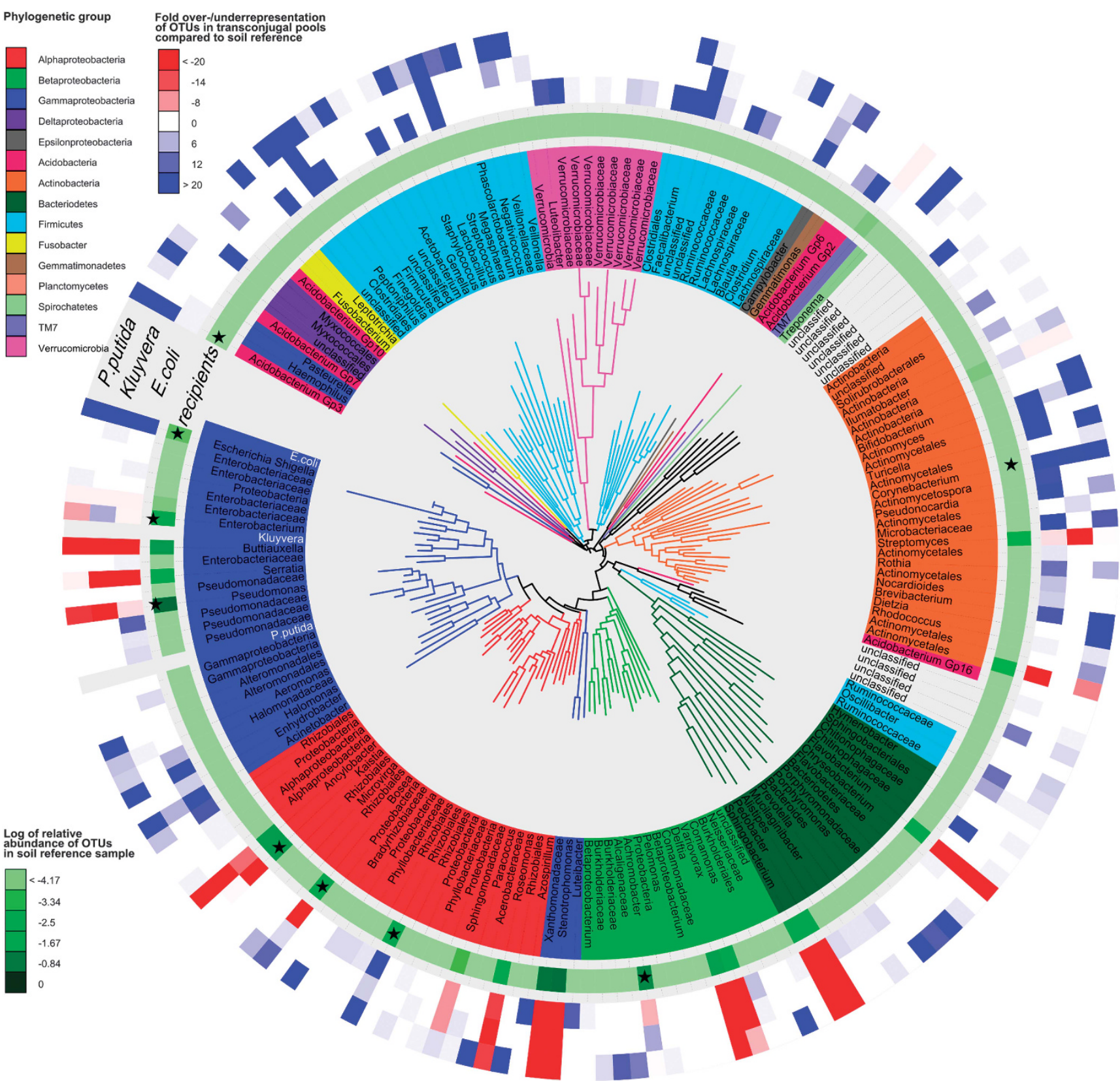

Figure 5 Phylogenetic tree showing all identified transconjugant OTUs for the same plasmid (pKJK5) introduced through three different donor strains (P.putida, Kluyvera sp. and E. coli). Colors of the branches mark different phylogenetic groups. The three donor strains are shown in white letters in the trees. One green heatmap circle around the tree represents the log-transformed relative OTU abundance in the soil reference recipient community. Three heatmap circles in blue and red display the $x$-fold over- and underrepresentation of the OTU in the respective transconjugal pool in comparison with the abundance in the reference soil sample. Stars mark the shared (present in all three transconjugal pools) and abundant (present at more than $1 \%$ relative sequence abundance) transconjugant OTUs, which constitute the core super-permissive community fraction. Sample size was normalized to 30000 sequences per transconjugal pool.

potential of the RP4 conjugation system has therefore been known in artificial constructs under laboratory conditions, we are the first to prove that a large proportion of this transfer potential can be realized in nature. A similarly huge transfer potential is demonstrated for pKJK5, a plasmid closely related to RP4 belonging to the IncP-1e subgroup and for pIPO2tet, an IncPromA-type phylogenetically more distant, cryptic plasmid that merely seems to provide plasmid mobilization capability to its host (Figure 4). We thus show that a variety of broad host range plasmids can effectively be transferred to, and encoded genes can be expressed in large proportions of the bacterial tree of life, much beyond the limited transfer range identified so far (Shintani et al., 2014). The realized transfer range in the soil community under natural conditions might be even higher taking into account that Nycodenz 
extraction might not be able to recover all bacterial phyla from the soil sample (Holmsgaard et al., 2011). Of the total extractable soil microbial community, only the phyla Chloroflexi, Deinococcus-Thermus, Nitrospira and SR1 were not represented in the transconjugal pools in our experiments.

In particular, we identified transfer from the used Gram-negative donor strains to a wide variety of Gram-positive bacteria (Figures 4 and 5). Over 15 OTUs within the Actinobacteria phylum and more than 10 OTUs belonging to six different orders of Bacilli and Clostridia within the Firmicutes phylum were identified as transconjugants. Inter-Gram conjugal gene transfer has been shown with vectors consisting partly of the broad host range transfer machinery of RP4 recombined with the $s a c B$ gene from Gram-positive Bacillus subtilis (Schäfer et al., 1994), but has only exceptionally been identified in natural habitats (Musovic et al., 2006). Our observations suggest that it may be a more common process than previously considered.

Abundance in recipient community and phylogenetic distance to the donor do not explain the composition of transconjugal pools

In spite of the large diversity within the transconjugal pools, not all OTUs of the recipient community were represented in each pool and the relative abundance of OTUs in transconjugal pools was very heterogeneous. Our method cannot distinguish between original horizontal plasmid transfer events from subsequent vertical plasmid transfer through growth of transconjugants on the mating filter. Therefore, relative abundance in the transconjugal pools can be influenced by the relative growth rate of recipients. However, the fact that OTU abundance in the transconjugal pools is not explained by their abundance in the reference soil recipient community (Figures 4 and 5 and Supplementary Table 2) indicates that plasmid transfer occurs preferentially to some recipients and that transconjugal pools are not simply determined by the recipient's growth ability.

Next, we tested whether phylogenetic distance between donor and recipient, calculated based on the Sogin distance algorithm (Sogin et al., 2006), influenced the abundance of individual OTUs among the transconjugal pools. We found no significant correlation between phylogenetic distance to the donor and recipient frequency in the transconjugal pools $(P=0.09-0.94)$ for any of the donor plasmid combinations (Supplementary Figure 2). For example, the most abundant OTUs in soil that do not appear in the transconjugal pools (Supplementary Table 2) are Gammaproteobacteria; they display more than $90 \%$ 16S rRNA gene sequence similarity to the donor strains, whereas other OTUs with $<70 \%$ sequence similarity to donor cells, such as several members of the Flavobacterium phylum, did receive at least one of the plasmids. Transfer of an IncP-1 plasmid from E. coli to phylogenetically distant Flavobacteria was detected in soil microcosms (Pukall et al., 1996), indicating that transfer to distant nodes of the phylogenetic tree is not only possible, but also realized in undisturbed soil environments. In pure culture, permissiveness toward broad host range plasmids of isolates that are indistinguishable by 16S rRNA gene analysis can differ by more than 100fold (Heuer et al., 2010). Here we confirm that inferring plasmid uptake and transfer frequency cannot be predicted based on the phylogenetic identity of an OTU.

However, we confirm the role of donors in defining the plasmid transfer host range (De Gelder et al., 2005), and show that this effect is significant even for two donors belonging to the same family of Enterobacteriales (E. coli and Kluyvera sp.) and thus sharing a high genomic similarity. The reasons behind this are uncertain, but certain strains might have distinct abilities to achieve efficient cell-to-cell contact with a specific recipient, for example, through specific mating mediating pheromones (Hirt, 2002). Earlier studies have shown that plasmid exchange between two taxonomically different species can exceed intraspecies transfer frequencies (Bingle et al., 2003), proving that the regulatory interactions of donor, recipient and plasmid can influence transfer efficiency.

Similarly, three broad host range plasmids, all carried by the same $P$. putida strain, were transferred to distinct pools of recipients. Yano et al. (2013) hypothesized that genetic differences appearing among closely related IncP-1 plasmids through plasmid backbone evolution can result in significant diversities in host range efficiency without affecting their broad host range nature. Such backbone alterations exist between the IncP-1 $\alpha$ (RP4) and IncP-1e (pKJK5) core regulatory proteins such as KorB, TrfA, TrbA and Ssb (Bahl et al., 2007). Although these two plasmids are incompatible (both IncP-1), differences in gene silencing and expression of the different core proteins could explain the different transconjugal patterns. As already minor differences in regulation between two IncP-1 plasmids lead to distinct transconjugal pools, it is coherent that the unrelated transfer machinery of plasmid pIPO2tet caused significantly $(P<0.05)$ dissimilar transconjugal pools when compared with the IncP ones.

A core superpermissive community fraction dominates gene transfer

Out of 281 OTUs identified in the transconjugal pools with the three different broad host range plasmids and $P$. putida as donor, 74 OTUs were common to all three pools (Figure 6a). A similar observation (46 out of 279 OTUs shared) held when comparing the transconjugal pools for plasmid pKJK5 introduced via three different donors 
942

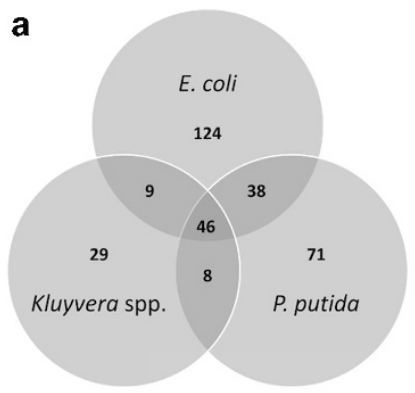

b
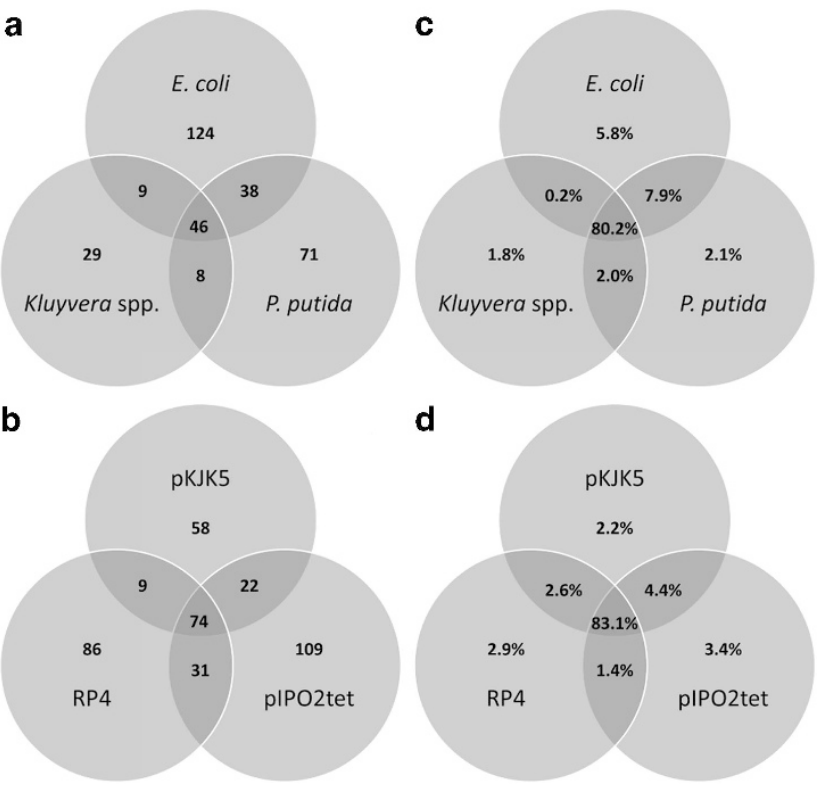

Figure 6 Venn diagram of transconjugal pools for plasmid pKJK5 transferred from three different donor strains (E. coli, P. putida and Kluyvera sp.) (a and c) and for three different plasmids (pKJK5, RP4 and pIPO2tet) (b and d) introduced through P. putida into the soil community. Venn diagrams are presented for OTU incidence (a and $\mathbf{b}$ ) and for OTU relative abundance (c and $\mathbf{d}$ ) $100 \%$ represents the total number of transconjugal sequences. OTUs were defined at $97 \%$ sequence similarity and sequence sample size was normalized to 30000 per transconjugal pool.

(Figure 6b). Therefore, the majority of transconjugant OTUs were only identified in single donorplasmid combinations. This might result from mating pair combinations that each favor or reduce gene transfer abilities (Bingle et al., 2003; Thomas and Nielsen, 2005; Yano et al., 2013).

Although only 74 and 46 OTUs are shared among the compared transconjugal pools, these OTUs represent over $80 \%$ of the transconjugal sequences (Figures 6c and d). This core super-permissive community fraction shared by all five transconjugal pools is able to take up diverse broad host range plasmids from diverse donor strains at high frequencies. The presence of this shared core in each analyzed transconjugal pool is the crucial discriminant that groups transconjugal pools apart from the original soil community (Figure 3). The core superpermissive community consists mainly of diverse Proteobacteria such as Enterobacteriales $(\gamma)$, Burkholderiales ( $\beta)$, Pseudomonadales $(\gamma)$ and Rhizobiales $(\alpha)$ (Figure 4). In addition, within this core super-permissive fraction, several OTUs that are rare in the recipient community $(<0.001 \%)$ are more than 20-fold overrepresented in transconjugal pools (Figure 4). The participation of these rare community members in gene transfer might have a crucial role in increasing the communal gene pool through rapid recombination with plasmids, as the rare biosphere can harbor a great reservoir of genes (Sogin et al., 2006).

\section{Medical relevance}

The large realized transfer potential of newly introduced plasmids in soil may be of medical importance. In recent EAHEC outbreaks in Germany, recombination of a pathogenic with the plasmid of a non-pathogenic E. coli strain increased the pathogenic potential to cause a deadly combination (Brzuszkiewicz et al., 2011). Soil-borne antibiotic resistance has been found to be shared with human pathogens (Benveniste and Davies, 1973; Forsberg et al., 2012). Several organisms among the identified transconjugants belong to groups known to contain opportunistic human pathogens, providing a direct link between the plasmid encoded mobile soil resistome and opportunistic pathogens. These groups include the proteobacterial Enterobacteria, Pseudomonas or Campylobacter, and also groups from other phyla such as Fusobacterium, Streptococcus and Staphylococcus, most of which are treated with antibiotic therapy. Especially the acquisition of new antibiotic resistance genes through plasmid-mediated gene transfer may push the pathogenic potential of Staphylococcus, originating from rapid evolution of virulence and drug resistance (Holden et al., 2004), even further.

The observed transfer of broad host range IncP-1type plasmids between Gram-negative and Grampositive bacteria might lead to a reassessment of the potential of soil bacterial communities to spread antibiotic resistance genes. Indeed, Actinobacteria, the origin of many soil-borne resistance genes (D'Costa et al., 2006), which are sometimes identified in clinical isolates of Gram-negative antibioticresistant bacteria (Benveniste and Davies, 1973), are frequent among the transconjugants we identified. Broad host range plasmids of the IncP-1 and IncPromA group can thus provide a direct link between diverse bacterial groups. Especially, IncP$1 \varepsilon$ plasmids such as pKJK5 have been identified as vectors of antibiotic resistance genes transfer among Proteobacteria by additionally hosting class 1 integron gene cassettes (Heuer et al., 2012). These class 1 integrons may not only spread in their originally identified Gram-negative Enterobacteriaceae hosts but can also be found among many Gram-positive bacteria (Nandi et al., 2004). Here, we demonstrated a possible direct way of accession of these class 1 integrons in Gram-positive bacteria through IncP-1ع plasmid transfer from Proteobacteria.

\section{Ecological and evolutionary relevance}

Plasmid host range can be defined in several ways depending on the duration and intimacy of the considered plasmid-host relationship, including the transfer host range, the replication and maintenance host range, or the evolutionary host range (Suzuki et al., 2010). We show here that the immediate transfer range for IncP plasmids is much wider than previously reported, proving that in the absence of physical barriers to cell-to-cell contact, broad host 
range plasmids have a high likelihood to be hosted by very diverse bacteria, at least transiently.

However, comparative analysis of plasmid sequences has indicated that the evolutionary host range of IncP plasmids seems to be mostly limited to Proteobacterial classes (Suzuki et al., 2010). This suggests that these plasmids are not maintained long enough outside of this phylum to be significantly affected by non-Proteobacterial genomes. Long-term evolutionary adaptation of the plasmid backbone to the new host, as known for IncP plasmids (Norberg et al., 2011), might therefore also not take place. Poor maintenance of these plasmids in non-proteobacterial hosts is the likely bottleneck explaining the difference between the very wide realized transfer range and the narrower evolutionary range. Mating pair formation and conjugation systems in these plasmids are evolutionarily adapted to connect and span Gram-negative membranes. The observed transfer to Gram-positive bacteria might therefore become a dead end in many cases for Gram-negative associated plasmids if the Type IV coupling and secretion system cannot efficiently spread the plasmid to other neighboring bacteria. However, an actinobacterial Mycobacterium strain has been shown to host and transfer an IncP-type plasmid, indicating that maintenance and transfer is possible across the Gram border (Leão et al., 2013). Also, the transient presence of a plasmid can provide the new host with a punctual adaptive gene pool and result in a short-term, but highly significant, fitness gain. Accessory genes on plasmids are mostly arranged in transposons flanked by insertion sequence (IS) elements, which can recombine with the recipient bacterial chromosomes (e.g. class 1 integron of pKJK5) delivering packages of fitness altering DNA without the need for plasmid replication. Additionally, transient hosts can increase the transfer range further by allowing transfer to organisms that had a lower transfer potential from the original donor strain (Yano et al., 2013).

We show within a bacterial community that there is a high variability in permissiveness to broad host range plasmids that cannot be explained by the phylogeny of the potential recipient. The ability to take up diverse broad host range plasmids from different hosts at high frequencies as represented by the super permissive fraction of the community has not previously been described. We do not know if it is a strain-specific trait and how environmental conditions affect its manifestation. Also, we do not know to what extent the mating conditions used might have biased the observed pattern of superpermissive plasmid recipients. However, if strainspecific, these super-permissive strains would be expected to have a disproportionate role as central nodes in networks of lateral gene acquisitions (Popa et al., 2011). Most gene acquisitions occur between donors and recipients residing in the same habitat (Popa and Dagan, 2011), and while gene acquisition in nature mainly occurs within taxonomically homogeneous groups, the heterogeneous soil community provides a hot-spot for gene acquisition from phylogenetically distant groups (Рopa et al., 2011). In soil, a few strains build the core nodes of a heavily connected network of lateral gene acquisition (Popa et al., 2011), which could be a possible indication of being part of the super-permissive fraction. These species are mainly found within Enterobacteriales (Gammaproteobacteria), Burkholderiales (Betaproteobacteria) and Staphylococci (Bacilli), groups that contain most of our superpermissive OTUs. Finding the same group of bacteria as central nodes in lateral gene transfer networks (Popa et al., 2011) and as main contributors to plasmid flow in soil suggests that there is indeed a link between increased plasmid uptake ability and long-term gene acquisition potential.

\section{Conflict of Interest}

The authors declare no conflict of interest.

\section{Acknowledgements}

We thank SM Milani for assistance in FACS sorting and method development; J Magid for access to the CRUCIAL field plot. This work was funded by the Villum Kann Rasmussen Foundation Center of Excellence CREAM (Center for Environmental and Agricultural Microbiology).

\section{References}

Bahl MI, Hansen LH, Goesmann A, Sørensen SJ. (2007). The multiple antibiotic resistance IncP-1 plasmid pKJK5 isolated from a soil environment is phylogenetically divergent from members of the previously established alpha, beta and delta sub-groups. Plasmid 58: 31-43.

Bahl MI, Oregaard G, Sørensen SJ, Hansen LH. (2009). Construction and use of flow cytometry optimized plasmid-sensor strains. Methods Mol Biol 532: 257-268.

Benveniste R, Davies J. (1973). aminoglycoside antibioticinactivating enzymes in actinomycetes similar to those present in clinical isolates of antibiotic-resistant bacteria. Proc Natl Acad Sci USA 70: 2276-2280.

Bingle LEH, Zatyka M, Manzoor SE, Thomas CM. (2003). Co-operative interactions control conjugative transfer of broad host-range plasmid RK2: full effect of minor changes in TrbA operator depends on KorB. Mol Microbiol 49: 1095-1108.

Brown Kav A, Sasson G, Jami E, Doron-Faigenboim A, Benhar I, Mizrahi I. (2012). Insights into the bovine rumen plasmidome. Proc Natl Acad Sci USA 109: 5452-5457.

Brzuszkiewicz E, Thürmer A, Schuldes J, Leimbach A, Liesegang H, Meyer F-D et al. (2011). Genome sequence analyses of two isolates from the recent Escherichia coli outbreak in Germany reveal the emergence of a new pathotype: Entero-AggregativeHaemorrhagic Escherichia coli (EAHEC). Arch Microbiol 193: 883-891. 
D’Costa VM, McGrann KM, Hughes DW, Wright GD. (2006). Sampling the antibiotic resistome. Science 311: 374-377.

Dechesne A, Pallud C, Bertolla F, Grundmann GL. (2005). Impact of the microscale distribution of a Pseudomonas strain introduced into soil on potential contacts with indigenous bacteria. Appl Environ Microbiol 71: 8123-8131.

Excoffier L, Smouse PE, Quattro JM. (1992). Analysis of molecular variance inferred from metric distances among DNA haplotypes: application to human mitochondrial DNA restriction data. Genetics 131: 479-491.

Finley RL, Collignon P, Larsson DGJ, McEwen SA, Li X-Z, Gaze WH et al. (2013). The scourge of antibiotic resistance: the important role of the environment. Clin Infect Dis 57: 704-710.

Forsberg KJ, Reyes A, Wang B, Selleck EM, Sommer MOA, Dantas G. (2012). The shared antibiotic resistome of soil bacteria and human pathogens. Science 337: 1107-1111.

De Gelder L, Vandecasteele FPJ, Brown CJ, Forney LJ, Top EM. (2005). Plasmid donor affects host range of promiscuous IncP-1beta plasmid pB10 in an activatedsludge microbial community. Appl Environ Microbiol 71: $5309-5317$.

Grahn AM, Haase J, Bamford DH, Lanka E. (2000). Components of the RP4 conjugative transfer apparatus form an envelope structure bridging inner and outer membranes of donor cells: implications for related macromolecule transport systems. J Bacteriol 182: 1564-1574.

Halary S, Leigh JW, Cheaib B, Lopez P, Bapteste E. (2010). Network analyses structure genetic diversity in independent genetic worlds. Proc Natl Acad Sci USA 107: 127-132.

Hansen CHF, Krych L, Nielsen DS, Vogensen FK, Hansen LH, Sørensen SJ et al. (2012). Early life treatment with vancomycin propagates Akkermansia muciniphila and reduces diabetes incidence in the NOD mouse. Diabetologia 55: 2285-2294.

Heinemann JA, Sprague GF. (1989). Bacterial conjugative plasmids mobilize DNA transfer between bacteria and yeast. Nature 340: 205-209.

Heuer H, Binh CTT, Jechalke S, Kopmann C, Zimmerling U, Krögerrecklenfort E et al. (2012). IncP-1e plasmids are important vectors of antibiotic resistance genes in agricultural systems: diversification driven by class 1 integron gene cassettes. Front Microbiol 3: 2.

Heuer H, Ebers J, Weinert N, Smalla K. (2010). Variation in permissiveness for broad-host-range plasmids among genetically indistinguishable isolates of Dickeya sp. from a small field plot. FEMS Microbiol Ecol 73: 190-196.

Hirt H. (2002). In vivo induction of virulence and antibiotic resistance transfer in Enterococcus faecalis mediated by the sex pheromone-sensing system of pCF10. Infect Immun 70: 716-723.

Holden MTG, Feil EJ, Lindsay JA, Peacock SJ, Day NPJ, Enright MC et al. (2004). Complete genomes of two clinical Staphylococcus aureus strains: evidence for the rapid evolution of virulence and drug resistance. Proc Natl Acad Sci USA 101: 9786-9791.

Holmsgaard PN, Norman A, Hede SC, Poulsen PHB, Al-Soud WA, Hansen LH et al. (2011). Bias in bacterial diversity as a result of Nycodenz extraction from bulk soil. Soil Biol Biochem 43: 2152-2159.

Hong S-H, Bunge J, Jeon S-O, Epstein SS. (2006). Predicting microbial species richness. Proc Natl Acad Sci USA 103: 117-122.
Inoue D, Sei K, Soda S, Ike M, Fujita M. (2005). Potential of predominant activated sludge bacteria as recipients in conjugative plasmid transfer. J Biosci Bioeng 100: $600-605$.

Jain A, Srivastava P. (2013). Broad host range plasmids. FEMS Microbiol Lett 348: 87-96.

Joseph SJ, Hugenholtz P, Sangwan P, Osborne CA, Janssen PH. (2003). Laboratory cultivation of widespread and previously uncultured soil bacteria. Appl Environ Microbiol 69: 7210-7215.

Leão SC, Matsumoto CK, Carneiro A, Ramos RT, Nogueira CL, Lima JD et al. (2013). The detection and sequencing of a broad-host-range conjugative IncP-1 $\beta$ plasmid in an epidemic strain of Mycobacterium abscessus subsp. Bolletii. Ahmed N (ed). PLoS One 8: e60746.

Letunic I, Bork P. (2007). Interactive Tree Of Life (iTOL): an online tool for phylogenetic tree display and annotation. Bioinformatics 23: 127-128.

Levy SSB, Marshall B. (2004). Antibacterial resistance worldwide: causes, challenges and responses. Nat Med 10: S122-S129.

Lozupone C, Lladser ME, Knights D, Stombaugh J, Knight R. (2011). UniFrac: an effective distance metric for microbial community comparison. ISME J 5: 169-172.

Magid J, Luxhøi J, Jensen LS, Møller J. (2006). Establishment of a long-term field trial with urban fertilizers. In Bruun S, Raupp J, Pekrun C, Oltmanns M, Köpke U (eds.) Is Recycling of Nutrients from Urban Areas to Peri-Urban Organic Farms Feasible? ISOFAR Scientific SeriesKöster: Bonn, Germany, pp 59-78.

McKenzie GJ, Craig NL. (2006). Fast, easy and efficient: site-specific insertion of transgenes into enterobacterial chromosomes using Tn7 without need for selection of the insertion event. BMC Microbiol 6: 39.

Musovic S, Dechesne A, Sørensen J, Smets BF. (2010). Novel assay to assess permissiveness of a soil microbial community toward receipt of mobile genetic elements. Appl Environ Microbiol 76: 4813-4818.

Musovic S, Klümper U, Dechesne A, Magid J, Smets BF. (2014). Long-term manure exposure increases soil bacterial community potential for plasmid uptake. Environ Microbiol Rep 6: 125-130.

Musovic S, Oregaard G, Kroer N, Sørensen SJ. (2006). Cultivation-independent examination of horizontal transfer and host range of an IncP-1 plasmid among Gram-positive and Gram-negative bacteria indigenous to the barley rhizosphere. Appl Environ Microbiol 72: 6687-6692.

Nandi S, Maurer JJ, Hofacre C, Summers AO. (2004). Gram-positive bacteria are a major reservoir of Class 1 antibiotic resistance integrons in poultry litter. Proc Natl Acad Sci USA 101: 7118-7122.

Norberg P, Bergström M, Jethava V, Dubhashi D, Hermansson M. (2011). The IncP-1 plasmid backbone adapts to different host bacterial species and evolves through homologous recombination. Nat Commun 2: 268 .

Norman A, Hansen LH, Sørensen SJ. (2009). Conjugative plasmids: vessels of the communal gene pool. Philos Trans $R$ Soc Lond Ser B 364: 2275-2289.

Ochman H, Lawrence JG, Groisman EA. (2000). Lateral gene transfer and the nature of bacterial innovation. Nature 405: 299-304.

Popa O, Dagan T. (2011). Trends and barriers to lateral gene transfer in prokaryotes. Curr Opin Microbiol 14: 615-623. 
Popa O, Hazkani-Covo E, Landan G, Martin W, Dagan T. (2011). Directed networks reveal genomic barriers and DNA repair bypasses to lateral gene transfer among prokaryotes. Genome Res 21: 599-609.

Pukall R, Tschäpe H, Smalla K. (1996). Monitoring the spread of broad host and narrow host range plasmids in soil microcosms. FEMS Microbiol Ecol 20: $53-66$.

Samuels AL, Lanka E, Davies JE. (2000). Conjugative junctions in RP4-mediated mating of Escherichia coli. J Bacteriol 182: 2709-2715.

Schloss PD, Gevers D, Westcott SL. (2011). Reducing the effects of PCR amplification and sequencing artifacts on $16 \mathrm{~S}$ rRNA-based studies. PLoS One 6: e27310.

Schloss PD, Westcott SL, Ryabin T, Hall JR, Hartmann M, Hollister EB et al. (2009). Introducing mothur: open-source, platform-independent, communitysupported software for describing and comparing microbial communities. Appl Environ Microbiol 75: 7537-7541.

Schäfer A, Tauch A, Jäger W, Kalinowski J, Thierbach G, Pühler A. (1994). Small mobilizable multi-purpose cloning vectors derived from the Escherichia coli plasmids pK18 and pK19: selection of defined deletions in the chromosome of Corynebacterium glutamicum. Gene 145: 69-73.

Sengeløv G, Kristensen KJ, Sørensen AH, Kroer N, Sørensen SJ. (2001). Effect of genomic location on horizontal transfer of a recombinant gene cassette between Pseudomonas strains in the rhizosphere and spermosphere of barley seedlings. Curr Microbiol 42: 160-167.

Shintani M, Matsui K, Inoue J-I, Hosoyama A, Ohji S, Yamazoe A et al. (2014). Single-cell analyses revealed transfer ranges of IncP-1, IncP-7, and IncP-9 plasmids in a soil bacterial community. Appl Environ Microbiol 80: $138-145$.
Sogin ML, Morrison HG, Huber JA, Mark Welch D, Huse SM, Neal PR et al. (2006). Microbial diversity in the deep sea and the underexplored "rare biosphere". Proc Natl Acad Sci USA 103: 12115-12120.

Suzuki H, Yano H, Brown CJ, Top EM. (2010). Predicting plasmid promiscuity based on genomic signature. J Bacteriol 192: 6045-6055.

Sørensen SJ. (1993). Transfer of plasmid RP4 from Escherichia coli K-12 to indigenous bacteria of seawater. Microb Rel 2: 135-141.

Sørensen SJ, Bailey M, Hansen LH, Kroer N, Wuertz S. (2005). Studying plasmid horizontal transfer in situ: a critical review. Nat Rev Microbiol 3: 700-710.

Thomas CM, Nielsen KM. (2005). Mechanisms of, and barriers to, horizontal gene transfer between bacteria. Nat Rev Microbiol 3: 711-721.

Wang Q, Garrity GM, Tiedje JM, Cole JR. (2007). Naive Bayesian classifier for rapid assignment of rRNA sequences into the new bacterial taxonomy. Appl Environ Microbiol 73: 5261-5267.

Waters VL. (2001). Conjugation between bacterial and mammalian cells. Nat Genet 29: 375-376.

Wolk CP, Vonshak A, Kehoe P, Elhai J. (1984). Construction of shuttle vectors capable of conjugative transfer from Escherichia coli to nitrogen-fixing filamentous cyanobacteria. Proc Natl Acad Sci USA 81: 1561-1565.

Yano H, Rogers LM, Knox MG, Heuer H, Smalla K, Brown CJ et al. (2013). Host range diversification within the IncP-1 plasmid group. Microbiology 159: 2303-2315.

Yue JC, Clayton MK. (2005). A similarity measure based on species proportions. Commun Stat 34: 2123-2131.

Zhaxybayeva O, Doolittle WF. (2011). Lateral gene transfer. Curr Biol 21: R242-R246.

Zhu Y-G, Johnson TA, Su J-Q, Qiao M, Guo G-X, Stedtfeld RD et al. (2013). Diverse and abundant antibiotic resistance genes in Chinese swine farms. Proc Natl Acad Sci USA 110: 3435-3440.

Supplementary Information accompanies this paper on The ISME Journal website (http://www.nature.com/ismej) 